\title{
Usefulness of analytic tests for the diagnosis of cow's milk protein allergy
}

\author{
M. Cristina Díaz ${ }^{a}(\mathbb{D})$, Alberto J. Lavrut ${ }^{a}$ (D) Pablo Slullitel $^{a}(\mathbb{D})$, M. Verónica Souza ${ }^{a}$ (D)
}

\begin{abstract}
Introduction. Cow's milk protein allergy is the most common food allergy among children. It can be diagnosed based on a guided history taking and using an oral food challenge (OFC), serum specific immunoglobulin E levels (sIgE), and skin prick tests (SPT). However, it is difficult to establish their diagnostic performance in the local population. Our objective was to assess the usefulness of tests used to diagnose cow's milk protein (CMP) allergy in the studied population. Population and methods. Retrospective analysis of data from patients seen at the Unit of Allergy of a tertiary care pediatric hospital between 2015 and 2018. SPT and SIgE tests were done for milk, alpha-lactalbumin, beta-lactoglobulin, and casein, followed by an OFC, and the diagnostic usefulness of each test, as well as their combination, was established.
\end{abstract}

Results. The tests of 239 patients were assessed. OFC was performed at the hospital in $54.8 \%$ of cases, via a rechallenge test at home in $35.5 \%$, and through CMP intake by the mother in $9.6 \%$. The highest specificity was observed with the casein SPT $(96.7 \%$; $95 \%$ confidence interval [CI]: 90.8-99.3) and the highest sensitivity, with the 4-allergen SPT and SIgE combination (55.3\%; 95\% CI: 45.7-64.6).

Conclusions. The study established the diagnostic usefulness of SPT and SIgE in the studied population.

Key words: milk hypersensitivity, diagnostic tests, predictive value of tests.

http: / / dx.doi.org/10.5546/ aap.2022.eng.21

a. Unit of Allergy, Hospital General de Niños "Pedro de Elizalde", Autonomous City of Buenos Aires, Argentina.

E-mail address:

M. Cristina Díaz:

dramcdiaz@hotmail. com

Funding:

None.

Conflict of interest:

None.

Received: 11-11-2020

Accepted: 7-5-2021

\section{INTRODUCTION}

The worldwide prevalence of food allergy has increased, ${ }^{1}$ both in developed and developing countries. ${ }^{2}$ This is specially problematic among children and affects their quality of life. ${ }^{3}$ Worldwide, between 220 and 250 million people may have food allergy. ${ }^{4}$

Cow's milk protein allergy (CMPA) is the most common food allergy during childhood, ${ }^{5}$ with a prevalence between 0.5 and $3 \%$ in developed countries. ${ }^{3,5}$ A study conducted in Argentina reported an $0.8 \%$ prevalence. $^{6}$

CMPA is defined as an hypersensitivity response to cow's milk protein, resulting from exposure to these allergens intake. ${ }^{3}$ Typically, such reaction is mediated by immunoglobulin $\mathrm{E}$ ( $\mathrm{IgE}$ ); however, its assessment is complex due to other mechanisms, including nonIgE-mediated or mixed reactions and non-immune mechanisms. ${ }^{3,7}$

CMPA diagnosis is based on careful assessment, including laboratory tests, and a definite determination using a double-blind, placebo-controlled food challenge (DBPCFC), considered to be the gold standard test. ${ }^{3}$ In practice, an unblinded oral food challenge (OFC) may be considered an appropriate option to DBPCFC. However, they are both costly in terms of time and resources and pose a risk for anaphylaxis. $3,8,9$

Skin prick tests (SPT) and serum specific IgE (sIgE) may be useful to diagnose CMPA, together with symptomatology.

However, their usefulness has not been clearly determined and varies depending on the different publications $\mathrm{s}^{10-17}$ and, to the authors' 
knowledge, there are no articles about the diagnostic usefulness of these tests in Argentina. ${ }^{5}$

\section{OBJECTIVE}

To assess the usefulness of tests used to diagnose to CMPA in the studied population.

\section{POPULATION AND METHODS \\ Data collection}

The medical records of the hospital's Unit of Allergy were digitized in Excel® and assessed for suspected CMPA in the 2015-2018 period, for a total of 623 records. This was a cross-sectional, retrospective study submitted to and approved by the hospital's Ethics Committee.

The variables included the following data: date of birth and first consultation; symptoms at onset; development of symptoms; test conduct, date, and results; feeding received throughout the different stages of life, and patient follow-up.

Patients in whom an OFC -considered the standard for diagnosis- $w$ as recorded were selected for analysis.

Urticaria, angioedema, anaphylaxis, rhinitis, recurrent obstructive bronchitis, asthma, and vomiting were considered a group of IgEmediated symptoms.

\section{Unit}

This is a public children's hospital located in the south of the Autonomous City of Buenos Aires, Argentina.

During the study period, a total of 353766 first-time consultations were made, with an annual average of 88442 consultations. In the same period, the Unit of Allergy received 5024 first-time consultations, with an annual average of 1256 consultations.

\section{Diagnostic algorithm}

The hospital's Unit of Allergy assesses suspected cases of CMPA in accordance with the DRACMA guidelines, ${ }^{7,18,19}$ as per the algorithm shown in Figure 1. It includes an ad hoc medical record designed to collect systematic and comprehensive information to reflect the guidelines (Figure 2).

\section{Diagnostic tests}

SPT: the following commercial allergens (Q alergia) were used: whole milk, alphalactalbumin (alpha), beta-lactoglobulin (beta), casein, with negative (saline solution) and positive controls (histamine). Disposable, single use, sterile lancets (Morrow-Brown model) were used for the procedure. Results were reviewed at 15 minutes. A wheal diameter $\geq 3 \mathrm{~mm}$ was considered positive.

sIgE: the Allergen ${ }^{\circledR}$ method is based on a capture enzyme-linked immunosorbent assay (ELISA) that uses a solid phase (strips of wells coated with human anti-IgE antibodies) for all tests with the different allergens (milk, alpha, beta, casein) and calibration curves. Results $>0.35 \mathrm{kU} / \mathrm{L}$ were considered detectable.

OFC: a previous 4-week period of strict elimination diet without CMP was required. Patients were assessed for CMPA, regardless of symptoms.

In the case of children who were exclusively breastfed, their mother's diet carefully excluded any CMP product and a calcium supplement was added.

In patients with exclusive formula-feeding or mixed-feeding, the use of a free amino acid formula was indicated to ensure absolute elimination of CMP. A weekly clinical control was conducted.

The diagnosis of CMPA was disregarded in patients who did not show improvement.

OFC was done in patients who showed a significant symptom improvement after the 4-week period. It consisted in an oral challenge with CMP while the patient was hospitalized at the day hospital in a room where oxygen, a laryngoscope, and anaphylaxis medicine (epinephrine injection, antihistamines, corticosteroids, bronchodilators, saline solution) were available and where a venous line was ready to administer drugs.

Oxygen saturation, heart rate, respiratory rate, and symptoms at initiation and after each dose up-titration were monitored.

The initial dose was $20 \mathrm{~mL}$, which was doubled every 20 minutes until reaching a dose adequate for age, weight, and nutritional status.

The test was stopped once symptoms developed; the necessary treatment actions were taken and diagnosis was confirmed.

If no symptoms appeared in the 2 hours after the last administration, the patient was discharged with indications for feeding with $\mathrm{CMP}$, warning signs, and outpatient follow-up at 24 and 48 hours and weekly for a month. If symptoms did not occur, CMPA was ruled out; otherwise, when symptoms occurred again, CMP was eliminated from the diet and diagnosis was confirmed (Figure 1). 


\section{Statistical analysis}

Variables for the 4-allergen SPT combination (combined SPT) and for the 4-allergen SIgE combination (combined sIgE) were developed; the result was considered positive if any assessment was positive, otherwise it was considered negative. The same was done for the combination of the 8 tests (combined SPT + sIgE).

For each test or combination, the area under the curve (AUC), sensitivity (Se), specificity $(\mathrm{Sp})$, positive predictive value (PPV), negative predictive value (NPV), positive likelihood ratio (LR+), and negative likelihood ratio (LR-) were estimated, considering the cut-off points described in the diagnostic test section.

Based on the prevalence of CMPA in the studied population as a pre-test probability, the post-test probabilities were estimated using the maximum LR+ and LR- obtained. Based on these values, Fagan's nomograms were also plotted. ${ }^{20}$

The Optimal Cutpoints and Uncertain Interval packages, part of the R software ${ }^{\circledR}$, version 3.6.1, were used.

\section{RESULTS}

Out of 623 medical records collected, 239 were selected because the performance of an OFC was verified. Among these, 126 corresponded

FIGURE 1. Diagnostic algorithm for the assessment of cow's milk protein allergy used in the Unit of Allergy of Hospital Elizalde according to the DRACMA guidelines

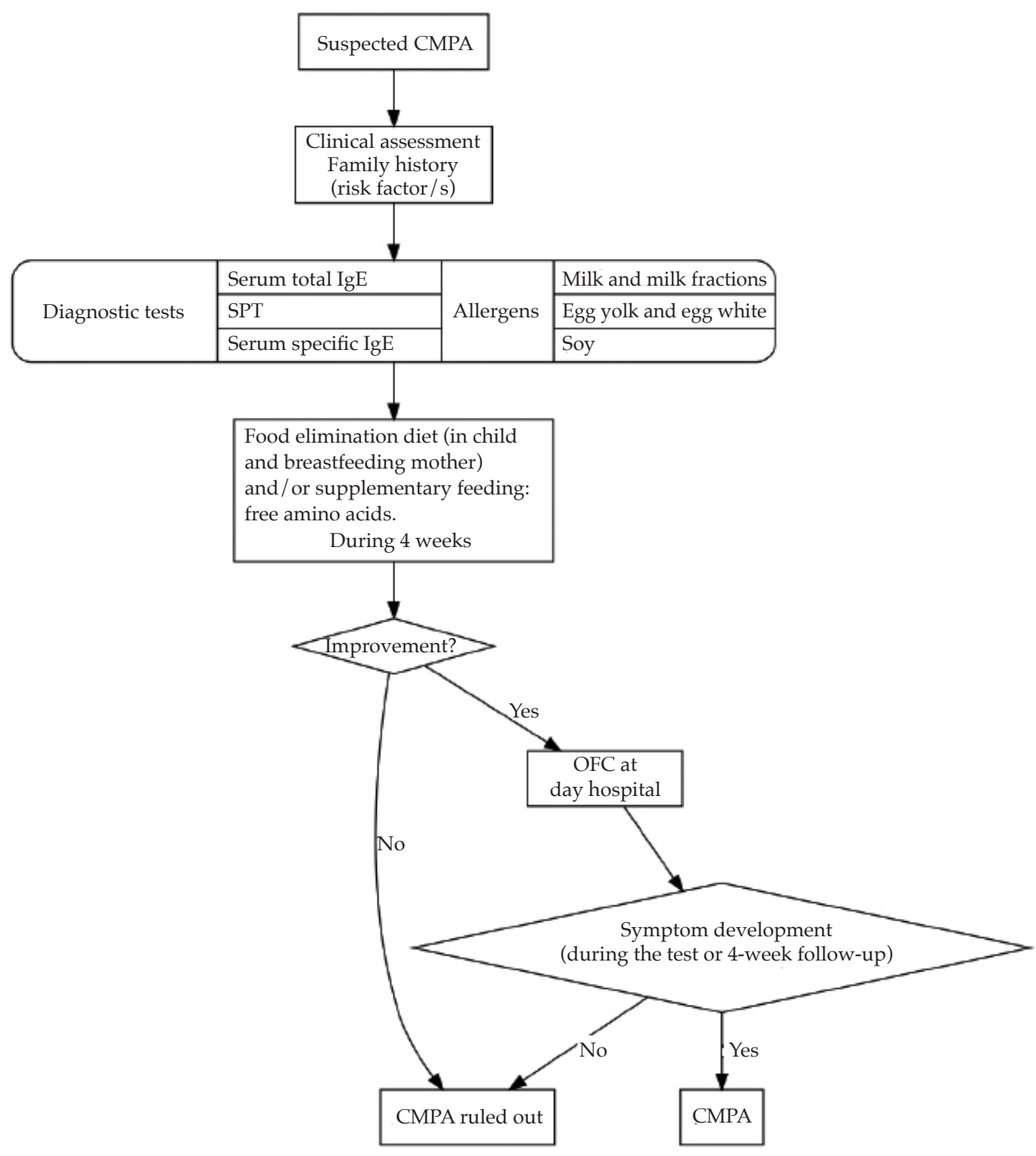

IgE: immunoglobulin E; SPT: skin prick test; OFC: oral food challenge; CMPA: cow's milk protein allergy. 
FIGURE 2. Ad hoc sheet designed and used by the hospital's Unit of Allergy to assess patients suspected of CMPA

History of cow's milk protein allergy (CMPA)

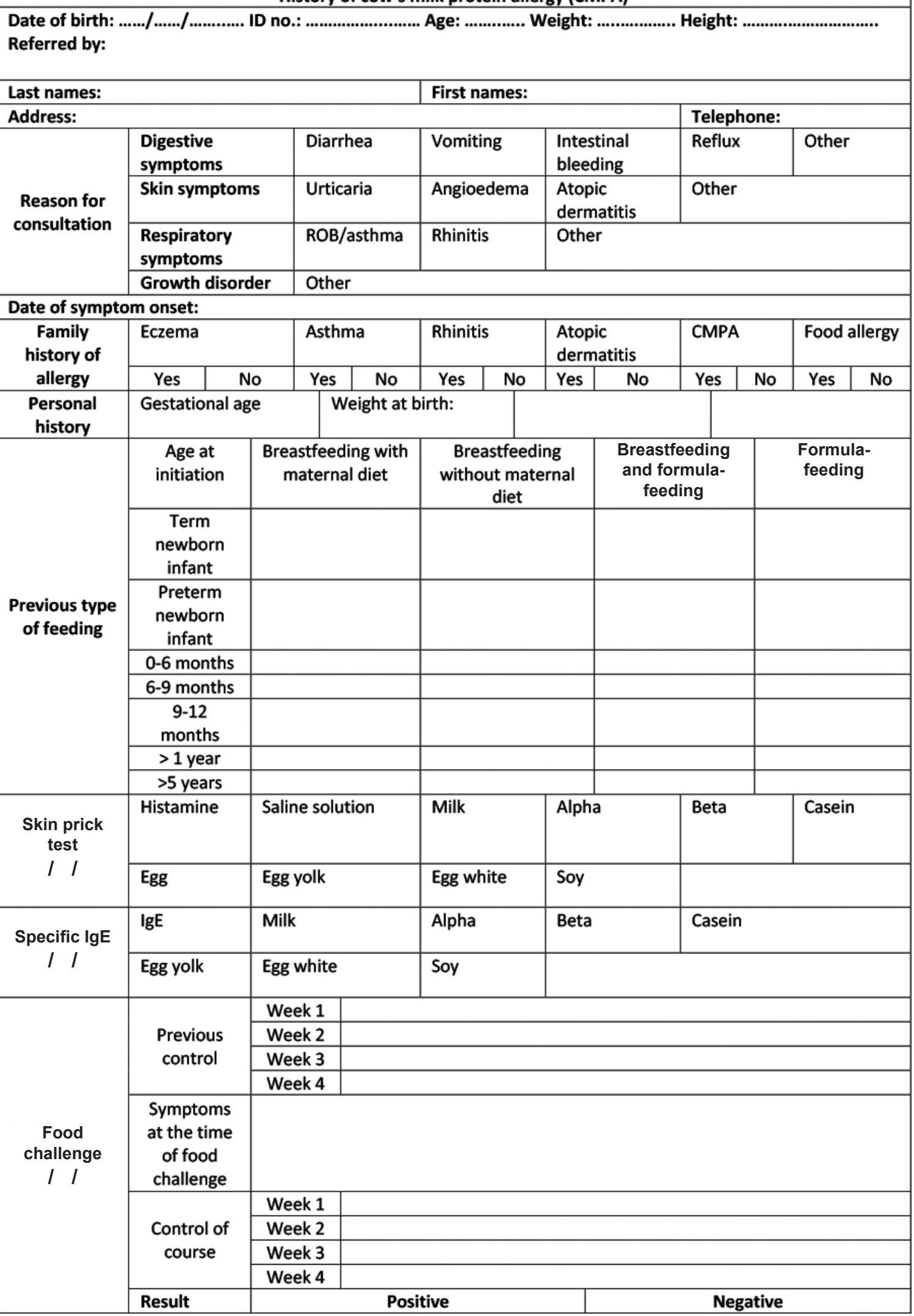


to patients with a definite diagnosis of CMPA, resulting in a prevalence of $52.7 \%(95 \%$ confidence interval [CI]: 46.4-59.0\%) in the studied population.

The mean age at symptom onset in these patients was 9.1 months and their median age was 5 months ( $1^{\text {st }}$ quartile $=1.6 ; 3^{\text {rd }}$ quartile $=10$ ). Figure 3 shows the distribution of symptoms.

The mean age at the time of the first consultation was 18.8 months and the median age was 10.4 months $\left(1^{\text {st }}\right.$ quartile $=5.4 ; 3^{\text {rd }}$ quartile $\left.=21\right)$.

Before the consultation, patients were receiving different types of feeding. Exclusive breastfeeding was the most common type (41\% of cases). The most common sequence before the consultation was breast milk followed by bottlefeeding (53\% of cases).

The mean follow-up of patients was 10.36 months, with a median follow-up of 6.1 months.

OFC was done at the hospital in $54.8 \%$ of patients. In $35.5 \%$, the occurrence of symptoms at home before rechallenge with CMP was considered a diagnosis. In $9.6 \%$, diagnosis was obtained by reintroducing CMP in the mother's diet, if the patient was exclusively breastfed.

Among positive tests, mediate reactions occurred in $10.5 \%$ of patients with IgE-mediated symptoms $(n=163)$ and in $14 \%$ of those with nonIgE-mediated symptoms $(\mathrm{n}=76)$.

Diagnostic usefulness was estimated in the 3 groups (total: 239 patients) and in each subgroup of IgE-mediated and non-IgE-mediated symptoms.

Results can be observed in Table 1. It is worth noting that the highest specificity was obtained in the casein SPT (96.7\% specificity) and the highest sensitivity, in the 4-allergen SPT and SIgE combination (55.3\% sensitivity).

When results are discriminated by IgEmediated symptoms, the highest specificity value was obtained in the casein SPT (specificity $=93.9 \%$ ) and the highest sensitivity, in the 4-allergen SPT and sIgE combination (sensitivity $=57.9 \%)$. Among patients with non-IgE-mediated symptoms, the highest specificity value was obtained in the beta sIgE (specificity $=97.9 \%)$ and the highest sensitivity, in the 4-allergen SPT and SIgE combination ( sensitivity $=42.1 \%)$. Figure 4 shows a plot comparing the different diagnostic performances, by type of presentation.

The LR+ and LR- values can be used to plot Fagan's nomograms, which allow to estimate the post-test probability value in a theoretical patient with a pre-test probability equal to the prevalence observed in the studied population. To develop the plot, the test results that obtained the highest $\mathrm{LR}+$ and the lowest LR- were selected (Figure 5).

In the case of the casein SPT, the probability of a patient being finally diagnosed with CMPA changes to $83.0 \%$, whereas the probability of ruling out CMPA changes to $49.6 \%$.

However, for the 4-allergen SPT and SIgE combination, these values were $63.5 \%$ and $43.5 \%$, respectively.

FIGURE 3. Bar chart showing the proportion of individual occurrence of each symptom in our patients. The dark gray bars indicate probably IgE-mediated symptoms

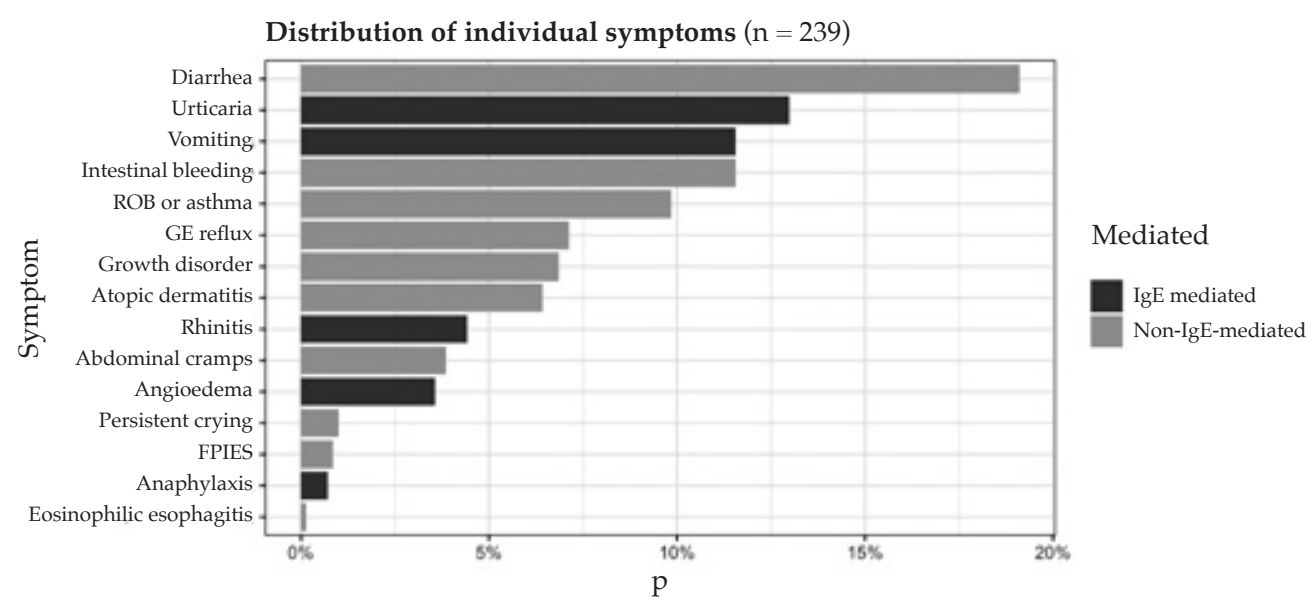

GE: gastroesophageal; ROB: recurrent obstructive bronchitis; FPIES: food protein induced enterocolitis syndrome; IgE: immunoglobulin E. 


\section{DISCUSSION}

In general, results showed high specificity and low sensitivity values. This indicates that the tests may be more appropriate to confirm a diagnosis than to screen for CMPA. ${ }^{21}$

The highest specificity values were associated with the use of casein as an allergen, both SIgE and SPT (94.1\% and 96.7\%, respectively).

The highest sensitivity values were obtained with the combination of at least 1 positive result among the 8 tests (55.3\% sensitivity) and, when considered individually, those that used milk as an allergen $(\mathrm{SPT}=34.7 \%$, sIgE $=24.6 \%$ ).

In general, when tests are analyzed by type of symptoms (IgE-mediated and non-IgE-mediated), the highest sensitivity values were observed in the IgE-mediated group, with a higher PPV. The latter is related to a higher prevalence of CMPA in this group.

The high prevalence observed here is explained by the fact that the study site is a referral hospital that receives a large number of patients referred by other units and facilities. This is in line with what has been reported in similar studies. $22-27$

Other authors have assessed the usefulness of CMPA diagnostic tests, but many times, a direct comparison is not possible because both antigens used and cut-off points for decision-making vary greatly. ${ }^{11}$ The cut-off points used here are based on the DRACMA guidelines. ${ }^{18}$

In comparison, the cut-off points proposed by other authors are much higher than those established in the guidelines. ${ }^{11}$

Our results are different from those obtained by other authors that used the same cut-off points, both with the $\operatorname{sigE} E^{22,26-31}$ and the SPT. ${ }^{10,22-27,29-32}$ However, in the studies that used the 4 antigens, the highest sensitivity was obtained with milk -as in our study-, whereas the remaining tests showed a higher specificity than sensitivity, both with the SPT ${ }^{10,29,32}$ and the sIgE. ${ }^{29}$ Furthermore, the greater specificity for SPT resulted in the same sense for casein. ${ }^{10,29,32}$

The differences mentioned above cannot be explained by the differences in cut-off points because they were the same in those studies.

The commercial reagents and equipment used in different studies vary greatly and, at least to the authors' knowledge, there is no standard that would allow to compare methodologies directly. This is a potential source of variability.

Another potential difference lies in the studied population because it included patients assessed with an OFC, regardless of their symptoms. However, other authors ${ }^{25,29}$ included only patients with symptoms suggestive of an IgE-mediated mechanism.

TABLE 1. Diagnostic performance values obtained with the skin prick test and specific immunoglobulin E, individually and with the different allergens combined

\begin{tabular}{|c|c|c|c|c|c|c|c|c|}
\hline Test & Cutoff point & AUC & Se $(\%, 95 \% \mathrm{CI})$ & $S p$ & PPV & NPV & LR+ & LR- \\
\hline Milk SPT & 3 & 0.61 & $34.7(26.3-44.0)$ & $86.4(78.2-92.4)$ & $75.0(63.0-81.5)$ & $53.0(43.0-68.2)$ & 2.55 & 0.76 \\
\hline Alpha SPT & 3 & 0.56 & $21.6(14.5-30.1)$ & $91.3(83.6-96.1)$ & $75.8(60.2-83.1)$ & $48.0(36.2-68.8)$ & 2.48 & 0.86 \\
\hline Beta SPT & 3 & 0.56 & $20.7(13.7-29.2)$ & $91.6(84.1-96.3)$ & $75.0(59.3-82.6)$ & $48.6(36.6-69.3)$ & 2.46 & 0.87 \\
\hline Casein SPT & 3 & 0.56 & $14.5(8.7-22.2)$ & 96.7 (90.8-99.3) & $85.0(65.2-90.5)$ & $47.1(33.3-81.5)$ & 4.46 & 0.88 \\
\hline Casein sIgE & 0.35 & 0.58 & $21.7(14.6-30.4)$ & $94.1(87.6-97.8)$ & $80.7(64.9-86.8)$ & $51.6(39.6-74.9)$ & 3.70 & 0.83 \\
\hline Beta sIgE & 0.35 & 0.57 & $19.3(12.5-27.7)$ & $94.1(87.5-97.8)$ & $78.6(61.9-85.5)$ & $50.8(38.2-74.3)$ & 3.25 & 0.86 \\
\hline Milk sIgE & 0.35 & 0.56 & $24.6(17.1-33.4)$ & $87.3(79.2-93.0)$ & $69.1(55.4-77.4)$ & $50.0(38.8-66.1)$ & 1.93 & 0.86 \\
\hline Alpha sIgE & 0.35 & 0.54 & $15.5(9.5-23.4)$ & $92.2(85.1-96.6)$ & $69.2(55.3-79.0)$ & $49.0(35.3-69.6)$ & 1.98 & 0.92 \\
\hline \multicolumn{9}{|l|}{ Combined } \\
\hline $\mathrm{SPT}+\operatorname{sIgE}$ & 1 & 0.60 & $55.3(45.7-64.6)$ & $64.6(53.3-74.9)$ & $68.5(57.6-76.2)$ & $51.0(41.4-62.9) 1.56$ & 1.56 & 0.69 \\
\hline Combined SPT & 3 & 0.61 & $39.7(30.7-49.2)$ & $83.1(73.7-90.2)$ & $75.4(63.6-81.9)$ & $51.4(41.6-66.5)$ & 2.35 & 0.73 \\
\hline Combined sIgE & 0.35 & 0.58 & $34.5(25.8-44.0)$ & $80.8(71.7-88.0)$ & $67.2(55.2-75.4)$ & $52.0(41.7-65.4)$ & 1.80 & 0.81 \\
\hline
\end{tabular}

CMPA: cow's milk protein allergy, SPT: skin prick tests, sIgE: serum specific immunoglobulin E, Se: sensitivity, Sp: specificity, NPV: negative predictive value, PPV: positive predictive value, LR: likelihood ratio, AUC: area under the curve,

Alpha: alpha-lactalbumin, Beta: beta-lactoglobulin. 
An interesting point that is worth noting is the exclusive use of formula based on amino acids during the elimination diet before the OFC. Such decision is based on the potential development of allergy to hydrolyzed formulas, which may occur in approximately $10 \%$ of individuals with CMPA. ${ }^{33}$ According to the preceding, and in accordance with the authors' experience, the use of formulas based on amino acids warrants a faster diagnosis and minimizes the impact on the quality of life of both the family and the patients who continue with allergy symptoms in spite of being fed with extensively hydrolyzed formulas. ${ }^{34}$

Lastly, it is important to note the population's genetic composition. As mentioned above, no other local study was found to allow the comparison with similar populations.

In addition, we should also note that the variability observed here may be due to the limitations implied by a retrospective study.

FIGURE 4. Proportion of each test's usefulness in terms of sensitivity, specificity, negative predictive value, and positive predictive value, by patients with or without probably IgE-mediated symptoms

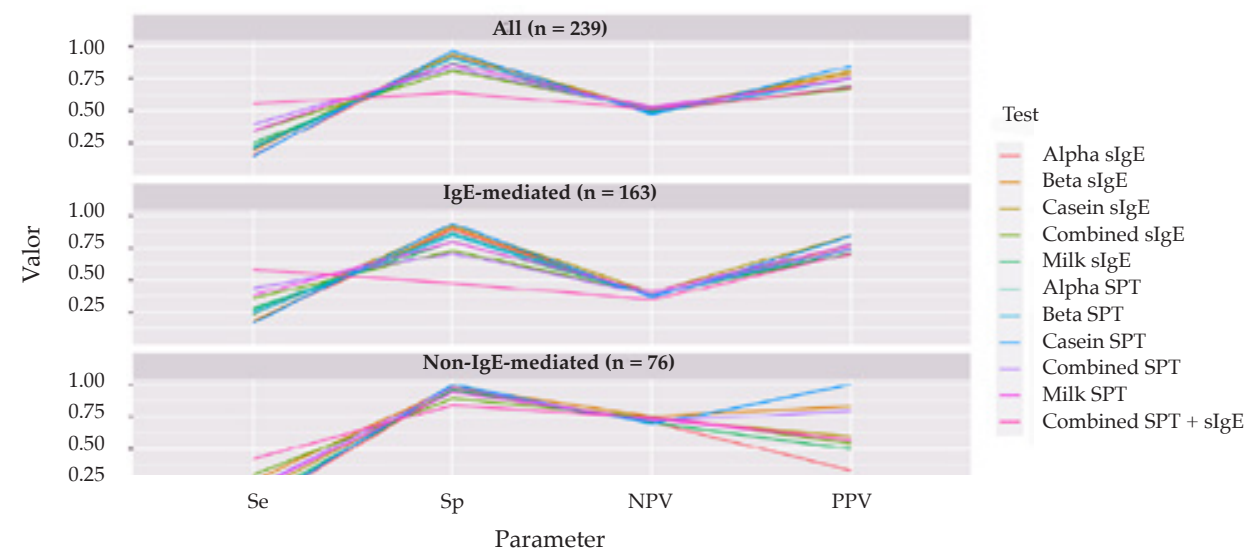

Se: sensitivity; Sp: specificity; NPV: negative predictive value; PPV: positive predictive value; sIgE: specific immunoglobulin E; Alpha: alpha-lactalbumin; Beta: beta-lactoglobulin; SPT: skin prick tests.

FIGURE 5. Fagan's nomogram showing the change in the probability of diagnosing cow's milk protein allergy, before and after the skin prick test using the antigen casein, regarding the prevalence of allergy at the time of the first assessment at the Unit of Allergy of Hospital Elizalde as the initial probability

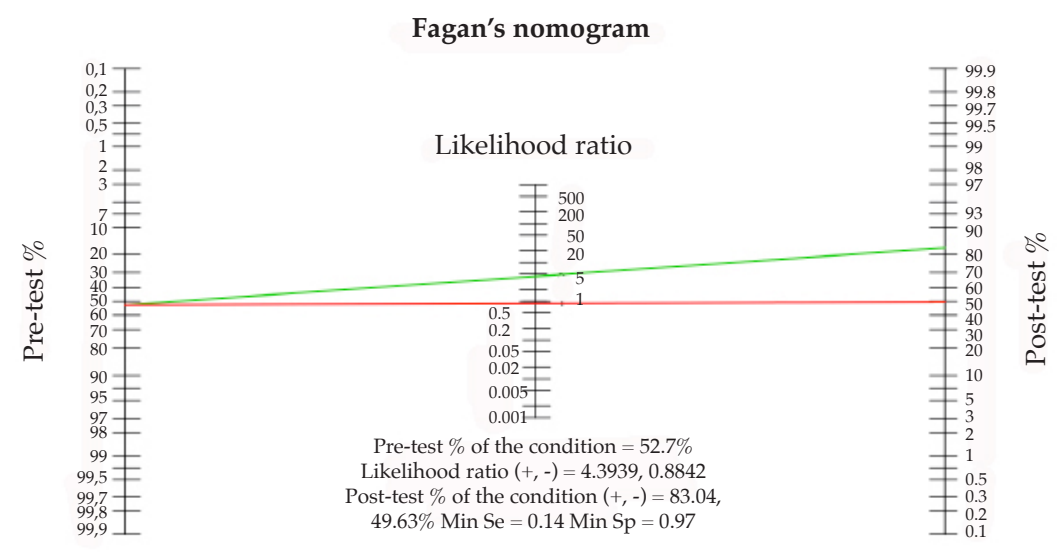

The green line accounts for the change in the probability of cow's milk protein allergy (CMPA) after the skin prick test, based on the use of a positive likelihood ratio, applied to a pre-test probability of $52.7 \%$, which corresponds to the baseline prevalence. 
Consistent with the higher specificity values observed, PPV were higher than NPV. The study by Calvani et al., ${ }^{10}$ assessed SPT with a cut-off point of $3 \mathrm{~mm}$, and found a better NPV for milk and a better PPV for casein. They also analyzed the combined SPT and found the best NPV. However, predictive values are correlated to the population prevalence ${ }^{35}$ and are not as useful for comparisons. These authors, ${ }^{10}$ based on considerations made by Sampson et al., ${ }^{27}$ used individual and combined SPT as predictors of OFC positivity, and found that SPT may be useful to avoid performing an OFC if predicted probability exceeded $95 \%$.

The observed likelihood ratios showed an intermediate or low discrimination power. LR+ values are higher, which is consistent with the better possibility of confirming a diagnosis. ${ }^{36}$ However, it is interesting to wonder how these tests may be used to make decisions in patients with varying pre-test probabilities, based on the symptoms at onset.

The authors consider that, once SPT and SIgE usefulness values are established in the studied population, these tests may help, together with symptoms that indicate a different probability, to make decisions, especially in relation to patients with IgE-like symptoms, and may render OFC unnecessary if the post-test probability exceeded the $95 \%$ threshold.

\section{CONCLUSIONS}

This study allowed to establish that, although SPT and SIgE showed a low sensitivity and NPV, they may be useful to make decisions in the studied population.

\section{REFERENCES}

1. Nehra M, Lettieri M, Dilbaghi N, Kumar S, Marrazza G. Nano-Biosensing Platforms for Detection of Cow's Milk Allergens: An Overview. Sensors (Basel). 2019; 20(1):32.

2. Prescott SL, Pawankar R, Allen KJ, Campbell DE, et al. A global survey of changing patterns of food allergy burden in children. World Allergy Organ J. 2013; 6(1):21.

3. Flom JD, SichererSH. Epidemiology of Cow's Milk Allergy. Nutrients. 2019; 11(5):1051.

4. Pawankar R, Canonica G, Holgate S, Lockey R. Allergic Diseases and Asthma: A Major Global Health Concern. Curr Opin Allergy Clin Immunol. 2012; 12(1):39-41.

5. Lifschitz C, Szajewska H. Cow's milk allergy: evidencebased diagnosis and management for the practitioner. Eur J Pediatr. 2015; 174(2):141-50.

6. Mehaudy R, Parisi C, Petriz N, Eymann A, et al. Prevalencia de alergia a la proteína de la leche de vaca en niños en un hospital universitario de comunidad. Arch Argent Pediatr. 2018; 116(3):219-23.

7. Fiocchi A, Brozek J, Schünemann H, Bahna SL, et al. World Allergy Organization (WAO) Diagnosis and Rationale for
Action against Cow's Milk Allergy (DRACMA) Guidelines. Pediatr Allergy Immunol. 2010; 21(Suppl. 21):105.

8. Huang F, Kim JS. IgE-Mediated Cow's Milk Allergy in Children. Curr Allergy Asthma Rep. 2012; 12(6):630-40.

9. Calvani M, Bianchi A, Reginelli C, Peresso M, Testa A. Oral Food Challenge. Medicina (Kaunas). 2019; 55(10):651.

10. Calvani M, Alessandri C, Frediani T, Lucarelli S, et al. Correlation between skin prick test using commercial extract of cow's milk protein and fresh milk and food challenges. Pediatr Allergy Immunol. 2007; 18(7):583-8.

11. Cuomo B, Indirli GC, Bianchi A, Arasi S, et al. Specific $\operatorname{IgE}$ and skin prick tests to diagnose allergy to fresh and baked cow's milk according to age: a systematic review. Ital J Pediatr. 2017; 43(1):93.

12. Fiocchi A, Bouygue GR, Restani P, Bonvini G, et al. Accuracy of skin prick tests in IgE-mediated adversereactions tobovine proteins. Ann Allergy Asthma Immunol. 2002; 89(6Suppl1):2632.

13. Mehl A, Niggemann B, Keil T, Wahn U, Beyer K. Skin prick test and specific serum IgE in the diagnostic evaluation of suspected cow's milk and hen's egg allergy in children: does one replace the other? Clin Exp Allergy. 2012; 42(8):1266-72.

14. Bellini F, Ricci G, Remondini D, Pession A. Cow's milk allergy (CMA) in children: identification of allergologic tests predictive of food allergy. Eur Ann Allergy Clin Immunol. 2014; 46(3):100-5.

15. Rancé F, Brondeau V, Abbal M. Use of prick-tests in the screening of immediate allergy to protein: 16 cases. Allerg Immunol (Paris). 2002; 34(3):71-6.

16. Sato S, Yanagida N, Ebisawa M. How to diagnose food allergy. Curr Opin Allergy Clin Immunol. 2018; 18(3):214-21.

17. Can C, Altinel N, Bülbül L, Civan HA, Hatipoğlu S. Clinical and Laboratory Characteristics of Patients with Food Allergy: Single-Center Experience. Sisli Etfal Hastan Tip Bul. 2019; 53(3):296-9.

18. Fiocchi A, Schunemann H, Ansotegui I, Assa'ad A, et al. The global impact of the DRACMA guidelines cow's milk allergy clinical practice. World Allergy Organ J. 2018;11(1):2.

19. Fiocchi A, Dahda L, Dupont C, Campoy C, et al. Cow's milk allergy: towards an update of DRACMA guidelines. World Allergy Organ J. 2016; 9(1):35.

20. Safari S, Baratloo A, Elfil M, Negida A. Evidence Based Emergency Medicine; Part 4: Pre-test and Post-test Probabilities and Fagan's nomogram. Emerg (Tehran). 2016; 4(1):48-51.

21. Ranganathan P, Aggarwal R. Common pitfalls in statistical analysis: Understanding the properties of diagnostic tests - Part 1. Perspect Clin Res. 2018; 9(1):40-3.

22. Saarinen KM, Suomalainen H, Savilahti E. Diagnostic value of skin-prick and patch tests and serum eosinophil cationic protein and cow's milk-specific IgE in infants with cow's milk allergy. Clin Exp Allergy. 2001; 31(3):423-9.

23. Verstege A, Mehl A, Rolinck-Werninghaus C, Staden U, et al. The predictive value of the skin prick test weal size for the outcome of oral food challenges. Clin Exp Allergy. 2005; 35(9):1220-6.

24. Keskin O, Tuncer A, Adalioglu G, Sekerel BE, et al. Evaluation of the utility of atopy patch testing, skin prick testing, and total and specific IgE assays in the diagnosis of cow's milk allergy. Ann Allergy Asthma Immunol. 2005; 94(5):553-60.

25. Ott H, Baron JM, Heise R, Ocklenburg C, et al. Clinical usefulness of microarray-based IgE detection in children with suspected food allergy. Allergy. 2008; 63(11):1521-8.

26. Roehr CC, Reibel S, Ziegert M, Sommerfeld C, et al. Atopy patch tests, together with determination of specific IgE levels, reduce the need for oral food challenges in children with atopic dermatitis. J Allergy Clin Immunol. 2001; 
107(3):548-53.

27. Sampson HA, Ho DG. Relationship between food-specific IgE concentrations and the risk of positive food challenges in children and adolescents. J Allergy Clin Immunol. 1997; 100(4):444-51.

28. Mehl A, Rolinck-Werninghaus C, Staden U, Verstege A, et al. The atopy patch test in the diagnostic workup of suspected food-related symptoms in children. J Allergy Clin Immunol. 2006; 118(4):923-9.

29. García-Ara C, Boyano-Martínez T, Díaz-Pena JM, MartínMuñoz F, et al. Specific IgE levels in the diagnosis of immediate hypersensitivity to cows' milk protein in the infant. J Allergy Clin Immunol. 2001; 107(1):185-90.

30. Majamaa H, Moisio P, Holm K, Kautiainen H, Turjanmaa K. Cow's milk allergy: diagnostic accuracy of skin prick and patch tests and specific IgE. Allergy. 1999; 54(4):346-51.

31. Comité Nacional de Alergia. Alergia alimentaria en pediatría: recomendaciones para su diagnóstico y tratamiento. Arch Argent Pediatr. 2018; 116(Supl 1):S1-19.

32. Onesimo R, MonacoS, Greco M, CaffarelliC, et al.Predictive value ofMP4 (Milk Prick Four), a panel of skin prick test for the diagnosis of pediatric immediate cow's milk allergy. Eur Ann Allergy Clin Immunol. 2013; 45(6):201-8.

33. AntunesJ, Borrego LM, Queiroz A, Chambel M, etal. Allergy to extensively hydrolysed formulas. Allergol Immunopathol (Madr). 2009; 37(5):272-4.

34. Stróżyk A, Horvath A, Meyer R, Szajewska H. Efficacy and safety of hydrolyzed formulas for cow's milk allergy management: A systematic review of randomized controlled trials. Clin Exp Allergy. 2020; 50(7):766-79.

35. Sackett DL, Straus SE, Richardson WS, Rosenberg W, Haynes RB. Evidence-Based Medicine: How to Practice and Teach EBM. $2^{\text {nd }}$ ed. New York: Churchill Livingstone; 2000.

36. McGee S. Simplifying Likelihood Ratios. J Gen Intern Med. 2002; 17(8):647-50. 